ARTICLE

Received 26 Nov 2014 | Accepted 13 Mar 2015 | Published 21 Apr 2015

DOI: $10.1038 /$ ncomms 7917

OPEN

\title{
Supramolecular-jack-like guest in ultramicroporous crystal for exceptional thermal expansion behaviour
}

Hao-Long Zhou ${ }^{1}$, Yue-Biao Zhang ${ }^{1,2}$, Jie-Peng Zhang ${ }^{1} \&$ Xiao-Ming Chen ${ }^{1}$

The dynamic behaviours of host frameworks and guest molecules have received much attention for their great relevance with smart materials, but little has been developed to control or understand the host-guest interplay. Here we show that the confined guest can utilize not only molecular static effects but also bulk dynamic properties to control the host dynamics. By virtue of the three-dimensional hinge-like framework and quasi-discrete ultramicropores, a flexible porous coordination polymer exhibits not only drastic guestmodulation effect of the thermal expansion magnitude (up to $422 \times 10^{-6} \mathrm{~K}^{-1}$ ) and even the anisotropy but also records positive/negative thermal expansion coefficients of $+482 /-218 \times 10^{-6} \mathrm{~K}^{-1}$. Moreover, single-crystal X-ray diffraction analyses demonstrate that the jack-like motion of the guest supramolecular dimers, being analogous to the anisotropic thermal expansion of bulk van der Waals solids, is crucial for changing the flexibility mode and thermal expansion behaviour of the crystal.

\footnotetext{
${ }^{1}$ MOE Key Laboratory of Bioinorganic and Synthetic Chemistry, School of Chemistry and Chemical Engineering, Sun Yat-Sen University, Guangzhou 510275, China. ${ }^{2}$ School of Physical Science and Technology, ShanghaiTech University, 100 Haike Road, Shanghai 201210, China. Correspondence and requests for materials should be addressed to J.-P.Z. (email: zhangjp7@mail.sysu.edu.cn).
} 
T he remarkable framework flexibility is one of the most important advantages of porous coordination polymers (PCPs) or metal-organic frameworks, which allows the host structures to be altered by external stimuli ${ }^{1}$. While chemical stimuli (mainly change of guest) have been extensively used as the driving forces ${ }^{2-5}$, physical stimuli such as light irradiation and temperature change have been scarcely explored ${ }^{6-10}$. Recently, flexible PCPs are emerging as an ideal platform for realizing abnormal temperature-induced structural dynamic behaviours, such as negative thermal expansion $(\mathrm{NTE})^{11-14}$ and very large thermal expansion (especially for $|\alpha|>100 \times 10^{-6} \mathrm{~K}^{-1}$; refs 15-20), which have great potential for compensation of the small positive thermal expansion (PTE, $0<\alpha<20 \times 10^{-6} \mathrm{~K}^{-1}$ ) of common solids, designing sensitive thermomechanical actuators, and so on $^{21-29}$. Besides framework flexibility and porosity, the designable framework connectivity of $\mathrm{PCP}^{30-32}$ offers an additional approach for engineering the magnitude and anisotropy of thermal expansion ${ }^{14-16,33,34}$. It should be noted that, for its response in different dimensions, anisotropic thermal expansion is attractive for microdevices and information materials ${ }^{29,35}$. More importantly, just like other physical properties $^{36-39}$, thermal expansion of PCP can be readily guest-dependent, albeit it is still very challenging ${ }^{16-19,40,41}$. As the most remarkable examples, Kepert and coworkers achieved isotropic PTE $\left(\alpha=+10.0 \times 10^{-6} \mathrm{~K}^{-1}\right)$ to NTE $\left(\alpha=-33.5 \times 10^{-6} \mathrm{~K}^{-1}\right)$ transition via guest removal in $\left[\mathrm{Cd}(\mathrm{CN})_{2}\right] \cdot x \mathrm{CCl}_{4}{ }^{41}$, and Barbour and coworkers showed that the PTE of $[\mathrm{Zn}(\mathrm{OH})($ niba $)]$.alcohol $\quad(\mathrm{Hniba}=4-(1 \mathrm{H}-$ naphtho[2,3-d]imidazol-1-yl)benzoic acid) can be increased by $\sim 90 \times 10^{-6} \mathrm{~K}^{-1}$ via changing the alcohol guest molecules ${ }^{18}$, in which the microporous structures have been demonstrated to be crucial for realizing the guest-modulation effect, since they maximize host-guest interaction around the guest molecules ${ }^{17,18,40,41}$.

On the other hand, the confinement effect of the porous host allows the formation of low-dimensional guest aggregations with abnormal/appealing physical properties ${ }^{17,39,42}$. The overwhelmingly large thermal expansion of the fluidic guest (usually beyond 100 times that of common solids) might be utilized to further drive the thermal expansion of the porous host. When the anisotropic structures of the host framework, pore environment and/or the low-dimensional guest aggregation are appropriately coupled, more exciting microscopic/macroscopic phenomenon could be expected. A key to these goals is to visualize, understand and control the delicate host-guest interplay under external stimulus, in which in situ single-crystal X-ray diffraction (SCXRD) is the most convenient, straightforward and reliable method ${ }^{43-45}$. Nevertheless, retaining the sample single-crystallinity after guest removal/absorption/ exchange and temperature cycling treatments is always a great challenge, and the extremely large mobility of guests also impedes determination of their positions ${ }^{16,17}$. Actually, even when the cross-section size of a one-dimensional channel is small enough to fit a single guest molecule well, the guest chain may still show liquid-like mobility ${ }^{17}$. To enable direct observation of thermal expansion of guest aggregations, discrete, isolated or $0 \mathrm{D}$ pores with suitable sizes for accommodation of two or a little bit more guest molecules should be optimal ${ }^{46,47}$. However, materials with such pores are generally difficult to change guests, preventing rational modulation of the physical property $20,48,49$.

In this work, we report a PCP possessing not only a threedimensional (3D) hinge-like structure with multimode flexibility but also quasi-0D ultramicropores suitable for adsorption and confinement of pairs of different amide-type guests. SCXRD studies of this material under temperature- and guest-stimuli reveal the largest NTE and PTE among crystalline solids to the best of our knowledge. We also report a drastic guest-modulation effect, and even a change of the main thermal expansion direction since the steric hindrance and thermal expansion effects of the guest dimers are large enough to alter the flexibility mode of the host framework (Fig. 1).

\section{Results}

Synthesis and structure of the porous crystal. Solvothermal reaction of $\mathrm{Cd}\left(\mathrm{NO}_{3}\right)_{2}, 3$-(pyridin-4-yl)benzoic acid (H34pba) and 4-(pyridin-4-yl)benzoic acid (H44pba) in mixed solvent ethanol/ water at $90^{\circ} \mathrm{C}$ afforded colourless needle-shaped crystals [Cd(34pba)(44pba)] (1, MCF-82). SCXRD analysis showed that $\mathbf{1}$ crystallizes in the monoclinic space group $P 2_{1} / c$ (Fig. 2, Supplementary Fig. 1, Supplementary Table 1 and Supplementary Data 1), containing one $\mathrm{Cd}^{2+}$ cation, one $34 \mathrm{pba}^{-}$ligand and one $44 \mathrm{pba}^{-}$ligand in its asymmetric unit. A pair of $\mathrm{Cd}^{2+}$ ions are bridged by two exobidentate carboxylate groups from two $34 \mathrm{pba}^{-}$ ligands and further coordinated by two chelating carboxylate groups from two $44 \mathrm{pba}^{-}$ligands and four monodentate pyridyl groups from two $34 \mathrm{pba}^{-}$and two $44 \mathrm{pba}^{-}$ligands to form a dinuclear $\mathrm{Cd}_{2}(\mathrm{RCOO})_{4}\left(\mathrm{~L}_{\mathrm{py}}\right)_{4}\left(\mathrm{~L}_{\mathrm{py}}=\right.$ pyridyl group $)$ unit, which interconnects with eight neighbours through four $34 \mathrm{pba}^{-}$and four $44 \mathrm{pba}^{-}$ligands to form a 3D coordination network with a uninodal 8-connected bcu topology (Fig. 2 and Supplementary Fig. 2$)^{50-52}$. Due to the presence of long bridging ligands, the crystal contains a quasi- $0 \mathrm{D}$ pore system, in which olive-like cavities with cross-sectional size of $6.0 \times 7.0 \times 9.7 \AA^{3}$ are interconnected through narrow necks (smaller than the diameter of a hydrogen atom) with diameter of $1.7 \AA$ along the $a$-axis.

Thermal expansion of the porous crystal. Variable-temperature SCXRD revealed giant temperature-induced crystal deformation property for 1 (Fig. 3, Supplementary Fig. 3, Supplementary Tables 1 and 2 and Supplementary Data 1 ). While the $a$ - and $c$-axes decrease by 2.2 and $1.6 \%$ from 112 to $300 \mathrm{~K}$, respectively, its $b$-axis has a significant increase of $9.0 \%$, giving rise to totally $5.8 \%$ increase of the unit-cell volume. Concomitantly, its void ratio increases from 20.4 to $24.8 \%$. Such large temperatureinduced crystal deformation has not been observed in coordination networks ${ }^{15-19,25}$. Comparison of the single-crystal structures at 112 and $300 \mathrm{~K}$ showed that, similar to other flexible

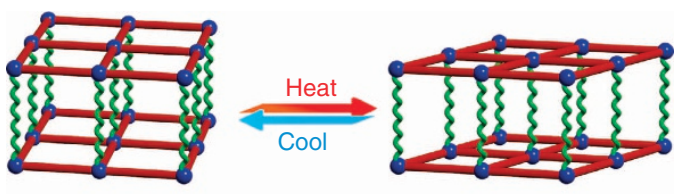

Guest restriction/expansion effect

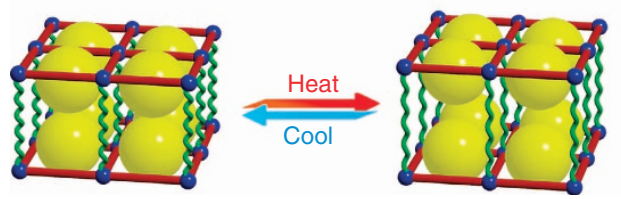

Figure 1 | Guest-controlled multimode framework flexibility. The red squares and green spires represent two possible responsive modes in a porous crystal encoded with multimode framework flexibility. Without guest, thermal expansion of the host framework is mainly determined by the hinge action across the red fences, which can be restricted by the steric hindrance effect of guest (yellow spheres). Further, the anisotropic thermal expansion effect of the guest dimers (significant change of separation between two yellow spheres), behaving like a jack, can force the host framework to deform in another direction along the green spires. 
PCPs, the framework distortions mainly arise from variation of the coordination angles rather than the coordination bonds (Supplementary Fig. 2 and Supplementary Tables 3 and 4). As defined by its bcu topology, the flexible 8-connected building unit can be regarded as a framework hinge and the whole framework can be considered as a $3 \mathrm{D}$ hinged rhombic fence consisting of many two-dimensional (2D) rhombic fences running across four crystallographic planes ${ }^{15,53}$. Although this framework topology is highly symmetric (cubic in its highest symmetric form), which allows the crystal to deform in any direction, the real deformation manner should depend on the anisotropy of the coordination network and pore system, as well as the distribution of the guest molecules. As the pores are running along the $a$-axis of 1 , it can be understood that the $3 \mathrm{D}$ coordination framework can distort easily on the $b c$-plane. Actually, there are 2D hinged fence-like

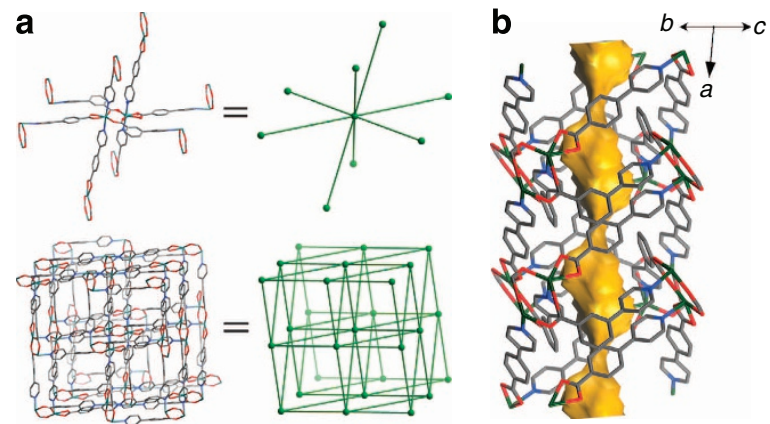

Figure 2 | X-ray single-crystal structure of the ultramicroporous framework. (a) Perspective view of 3D coordination framework of $\mathbf{1}$ along the $a$-axis (the aromatic rings of the $34 \mathrm{pba}^{-}$and $44 \mathrm{pba}^{-}$ligands are highlighted in blue and yellow, respectively). (b) Solvent-accessible pore surface structure of $\mathbf{1}$ viewed along the [011] direction, in which large cavities $\left(6.0 \times 7.0 \times 9.7 \AA^{3}\right)$ are interconnected by very narrow necks $(d \approx 1.7 \AA)$. motifs (consisting of dinuclear clusters and bent $34 \mathrm{pba}^{-}$ligands) running across the $b c$-plane, whose distortion causes the acute expansion of the $b$-axis and simultaneous contraction of the $c$-axis, when the temperature increases. The relative small contraction of the $c$-axis can be attributed to the nonplanar shape of the $b c$-plane hinged fence, which transfers some contraction effect to the $a$-axis.

Host-guest behaviour of the porous crystal. Gas adsorption measurements at low temperatures (Supplementary Fig. 4) showed that while 1 cannot adsorb $\mathrm{N}_{2}$ and Ar with kinetic diameters of 3.64 and $3.54 \AA$, respectively, at $77 \mathrm{~K}$, it can readily adsorb the smaller $\mathrm{O}_{2}(3.47 \AA)$ at the same condition. Nevertheless, the $\mathrm{O}_{2}$ isotherm exhibits obvious hysteresis and the saturated uptake $\left(32 \mathrm{~cm}^{3} \mathrm{~g}^{-1}\right)$ is significantly lower than the value empirically calculated from the crystal structure $\left(146 \mathrm{~cm}^{3} \mathrm{~g}^{-1}\right)$, indicating that the adsorption/desorption is very slow due to the high-energy barrier for gas diffusion in the quasi-0D ultramicropores. On the other hand, being similar with some flexible porous coordination polymers $3,16,1$ can reversibly adsorb/release $N, N$-dimethylformamide (DMF) and $N, N$-dimethylacetamide (DMA) vapour at room temperature to give [Cd(34pba)(44pba)].DMF (1.DMF) and [Cd(34pba)(44pba)]. DMA (1.DMA) without destroying the single-crystallinity (Supplementary Figs 5-7), indicating that the host framework possesses notable framework flexibility, especially for solvent molecules. SCXRD analyses of 1.DMF and 1.DMA at room temperature showed that they are isomorphic with $\mathbf{1}$. Their unit-cell volumes are only $1.4 \%$ and $2.5 \%$ larger than that of 1 (Supplementary Fig. 1, Supplementary Table 1 and Supplementary Data 1 ), which are relatively small among flexible $\mathrm{PCPs}^{2-4}$. The most important structural feature of 1.DMF and 1.DMA is that each cavity is occupied by a pair of DMF/DMA guest molecules arranged in a centrosymmetric fashion with their molecular planes parallel with each other. Due to the size a
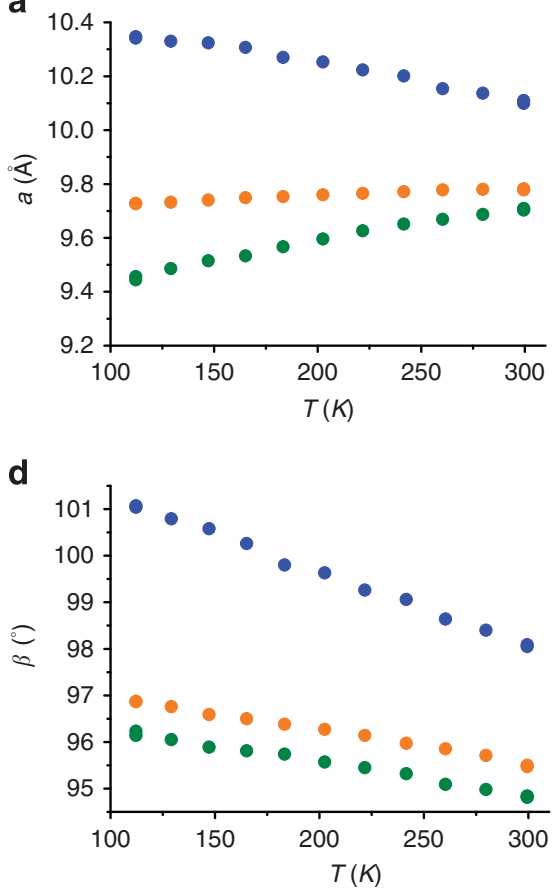

b
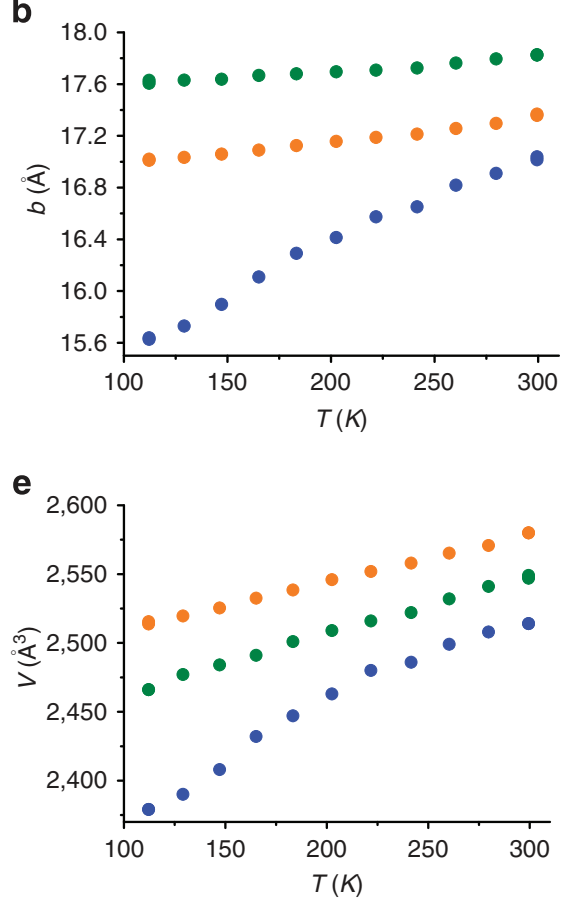

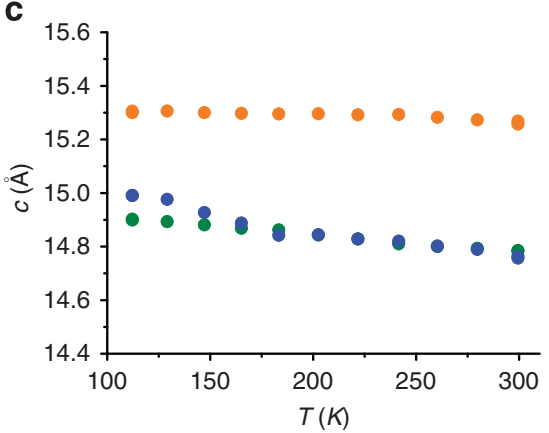

$\mathbf{f}$

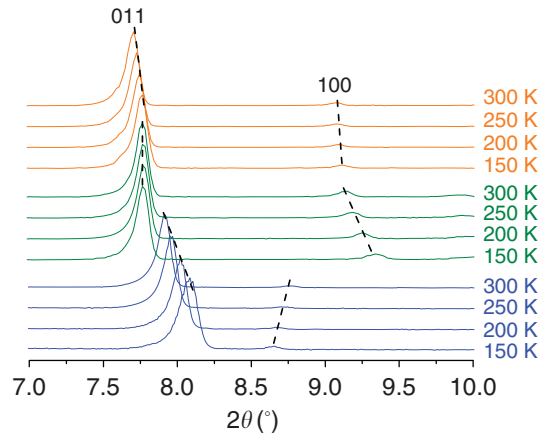

Figure 3 | Guest-dependent and guest-induced crystal deformation. (a-e) Temperature-dependent unit-cell parameters of 1 (blue), 1.DMF (green) and 1.DMA (orange) determined by SCXRD. (f) PXRD patterns of 1 (blue), 1.DMF (green) and 1.DMA (orange). 
difference of the guests, the DMF and DMA supramolecular dimers are distinct in their configurations ${ }^{54,55}$. Specifically, the molecular plane of DMA has an inclined angle of $40.0^{\circ}$ with the $b c$-plane, while that of DMF is only $17.3^{\circ}$, because the small pore forces the larger guest molecule to rotate to avoid significant steric hindrance. As a result of these molecular orientations, the DMF dimer exhibits much larger overlap as compared with those in the DMA dimer (Supplementary Fig. 8 and Supplementary Table 5). Consequently, adjacent DMA dimers form weak interaction $\left(\mathrm{CH}_{3} \cdots \mathrm{CH}_{3} 4.15 \AA\right)$, while the DMF dimers are well isolated from each other (shortest separation O… $5.71 \AA$ ).

Supramolecular-jack-like motion of the guest aggregations. Variable-temperature powder X-ray diffraction (PXRD) showed that (Fig. 3), while the (011) peaks of the three compounds gradually move to lower angles in different extents during temperature increase, the (100) peaks of 1 and $\mathbf{1} \cdot \mathrm{DMF} / \mathbf{1} \cdot \mathrm{DMA}$ move in different directions, indicating that the thermal responses of their $a$-axes contrast with each other. SCXRD revealed that (Fig. 3, Supplementary Fig. 3, Supplementary Tables 1 and 2 and Supplementary Data 1), while the $a$-axis of 1 decreases by $2.2 \%$ from 112 to $300 \mathrm{~K}$, those of $\mathbf{1} \cdot \mathrm{DMF}$ and $\mathbf{1} \cdot \mathrm{DMA}$ increase by 2.8 and $0.7 \%$, respectively. Moreover, the unit-cell volume and the $b$-/c-axis of 1 changed more largely than those of $1 \cdot \mathrm{DMF}$ and 1.DMA.

The restricted hinge motion of the $b c$-plane can be explained by the intrinsic steric hindrance effects of the guest molecules. When the quasi- $0 \mathrm{D}$ cavities, that is, the apertures of the $2 \mathrm{D}$ hinged fences on the $b c$-plane, are filled by guest molecules, the flexibility of the hinged fence is reduced, giving smaller temperature-induced deformation on the $b$-/c-axis. Though DMF is smaller than DMA, the temperature-induced hinge motion on the $b c$-plane of $\mathbf{1} \cdot \mathrm{DMF}$ is the smallest one among the three materials, because the DMF molecules are almost parallel with the $b c$-plane, which exhibit strong steric hindrance effect with the host framework and restrain the hinge action across this plane (Supplementary Fig. 9).

More importantly, the reversed directions of changes of the $a$-axis can be assigned to the thermal expansion of the guest supramolecular aggregation (Fig. 4, Supplementary Figs 8 and 10 and Supplementary Table 6). In 1.DMF, the interplanar separation within a dimer significantly increases by $0.45 \AA$ from 112 to $300 \mathrm{~K}$, while this value is only $0.15 \AA$ in $\mathbf{1}$.DMA, which highlights that the thermal expansion of the guest aggregation mainly occurs at the directions of the supramolecular interactions, and the tighter supramolecular contacts between the guest molecules can more effectively response to their local thermal vibrations. Comparison of their temperature-dependent electron density maps can clearly illustrate dynamic behaviours of the guest molecules. On the other hand, the closest interdimer separation decreases by $0.09 \AA$ in $\mathbf{1}$.DMF but increases by $0.17 \AA$ in 1.DMA. Since the interdimer interaction is ignorable in the crystal 1.DMF, the separation does not expand with increasing temperature. Instead, the large thermal expansion within the DMF dimer reduces the interdimer separation. Nevertheless, the thermal expansion of the dimer mainly transfers to or expands the flexible host framework via host-guest interaction, so that the interdimer separation decreases by much $<0.45 \AA$. In the case of 1.DMA, the interdimer supramolecular contact is close enough to show thermal expansion, and the intradimer thermal expansion is not significant enough to compress the interdimer separation. As defined by the directions of the guest-guest interactions, the thermal expansions of the guests are highly anisotropic in the crystals, which is relatively parallel with the $a$-axis of $\mathbf{1} \cdot \mathrm{DMF}$ or has a considerable inclined angle with the $a$-axis of 1.DMA. Therefore, the $a$-axis of 1.DMA expands much less than
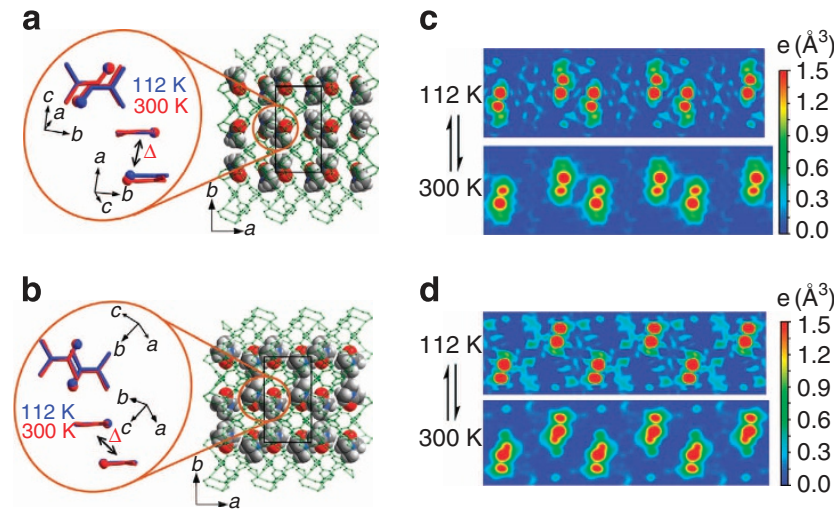

Figure 4 | Temperature-induced structural transformations of the guest supramolecular aggregations. (a,b) Crystal structures of (a) 1.DMF and (b) 1.DMA. The host frameworks and the guest molecules are shown in green stick and multicolour space-filling modes (carbon, grey; nitrogen, blue; oxygen, red; hydrogen atoms are omitted for clarity), respectively. The enlarged insets compare the relative positions of the two guest molecules (stick mode with oxygen atoms highlighted as spheres) within the supramolecular dimer at 112 (blue) and 300 (red) K, along two representative directions (perpendicular and parallel to the molecular planes). The double-headed arrows represent the vectors of the guestguest movements from 112 to $300 \mathrm{~K}$ within each dimer. (c,d) Temperaturedependent electron density maps of (c) 1.DMF and (d) 1.DMA.

1.DMF, since the thermal expansion of DMA supramolecular aggregations contribute to both the $a$ - and $b$-axes. What's more, the void volume of $\mathbf{1} \cdot \mathrm{DMF}$ (from 23.4 to $27.0 \%$ ) has a larger increase than that of 1.DMA (from 27.5 to $28.8 \%$ ), further indicating that the DMF dimer with much larger overlap can have stronger thermal expansion effect. While the static effects (that is, steric hindrance and supramolecular attraction to the host) of individual guest molecules have been observed to affect the thermal expansion behaviours of a few host frameworks ${ }^{18,40,41}$, this is the first structural evidence of thermal expansion of guest aggregations, which performs like supramolecular jacks to force the deformation of the host framework. It should be also mentioned that the relatively low crystallographic symmetry and suitable pore size (for a pair of amide molecules), as well as the multimode framework flexibility of $\mathbf{1}$ are decisive for the unambiguous observation of these interesting phenomena.

Principal axial thermal expansion of the crystal. Since 1, 1.DMF and 1.DMA crystallize in the monoclinic crystal system, the axial thermal expansion coefficients of the principal axes were calculated using the programme PASCal (Table 1 and Supplementary Table 7$)^{56}$. The principal $\mathrm{X}_{2}$-axis is the same as the original crystallographic $b$-axis, while the principal $\mathrm{X}_{1}$ - and $\mathrm{X}_{3}$-axes approximate the original crystallographic $a$ - and $c$-axes or the [101] and [-101] directions. All the unit-cell parameters of 1, 1.DMF and 1.DMA change linearly or approximately linearly against temperature, meaning that their axial thermal expansion coefficients are virtually constant in the measured temperature range. Such behaviour is critical for precise thermomechanical actuators and sensors. 1.DMF shows huge PTE along the $\mathrm{X}_{1}$-axis $\left(\alpha_{1}=+171 \times 10^{-6} \mathrm{~K}^{-1}\right)$ and moderate PTE and NTE along the $\mathrm{X}_{2^{-}}$and $\mathrm{X}_{3^{-}}$axes $\left(\alpha_{2}=+60 \times 10^{-6} \mathrm{~K}^{-1}\right.$, $\alpha_{3}=-56 \times 10^{-6} \mathrm{~K}^{-1}$ ), respectively. When DMF is replaced by DMA, the thermal expansion coefficients of the $\mathrm{X}_{1^{-}}$and $\mathrm{X}_{2}$-axes are approximately halved and doubled $\left(\alpha_{1}=+85 \times 10^{-6} \mathrm{~K}^{-1}\right.$, $\left.\alpha_{2}=+103 \times 10^{-6} \mathrm{~K}^{-1}\right)$, respectively, while that of the $\mathrm{X}_{3}$-axis is almost unchanged $\left(\alpha_{3}=-51 \times 10^{-6} \mathrm{~K}^{-1}\right)$. More remarkably, 


\begin{tabular}{|c|c|c|c|c|}
\hline Compound & $\begin{array}{c}\text { Principal } \\
\text { axis }\end{array}$ & Direction & $\left(\begin{array}{c}\alpha \\
\left(\times 10^{-6} K^{-1}\right.\end{array}\right)$ & $\begin{array}{c}\beta v_{v} \\
\left(\times 10^{-6} K^{-1}\right)\end{array}$ \\
\hline 1 & $\begin{array}{l}x_{1} \\
x_{2} \\
x_{3}\end{array}$ & $\begin{array}{l}\sim a \\
b \\
\sim c\end{array}$ & $\begin{array}{c}+61(1) \\
+482(12) \\
-218(3)\end{array}$ & $+319(13)$ \\
\hline 1.DMF & $\begin{array}{l}x_{1} \\
x_{2} \\
x_{3}\end{array}$ & $\begin{array}{l}\sim a \\
b \\
\sim c\end{array}$ & $\begin{array}{l}+171(4) \\
+60(3) \\
-56(2)\end{array}$ & $+175(2)$ \\
\hline 1.DMA & $\begin{array}{l}x_{1} \\
x_{2} \\
x_{3}\end{array}$ & $\begin{array}{l}\sim a \\
b \\
\sim c\end{array}$ & $\begin{array}{r}+85(1) \\
+103(4) \\
-51(3)\end{array}$ & $+138(2)$ \\
\hline
\end{tabular}

$\alpha$ and $\beta_{\vee}$ represent axial and volumetric thermal expansion coefficients, respectively.

although the PTE of the $\mathrm{X}_{1}$-axis of $1\left(\alpha_{1}=+61 \times 10^{-6} \mathrm{~K}^{-1}\right)$ is smaller than those of 1.DMF and 1.DMA, its $\mathrm{X}_{2^{-}}$and $\mathrm{X}_{3}$-axes exhibit giant PTE of $\alpha_{2}=+482 \times 10^{-6} \mathrm{~K}^{-1}$ and NTE of $\alpha_{3}=$ $-218 \times 10^{-6} \mathrm{~K}^{-1}$, respectively, which are much larger than for other known crystalline materials (Supplementary Table 8). It is noteworthy that the guest-modulation effect among 1, 1.DMF and 1.DMA are exceptionally enormous, which change the PTE and NTE magnitudes by up to $422 \times 10^{-6} \mathrm{~K}^{-1}$ and $167 \times 10^{-6} \mathrm{~K}^{-1}$, respectively. Also interestingly, the $\mathrm{X}_{2}$-axial PTE coefficient follows the order $\mathbf{1}>\mathbf{1} \cdot \mathrm{DMA}>\mathbf{1} \cdot \mathrm{DMF}$, while the $\mathrm{X}_{1}$-axial PTE coefficient follows the opposite order $\mathbf{1} \cdot \mathrm{DMF}>\mathbf{1} \cdot \mathrm{DMA}>\mathbf{1}$. Consequently, the thermal expansion coefficients of the $\mathrm{X}_{2^{-}}$and $\mathrm{X}_{3}$-axes of 1 are much larger than that of its $X_{1}$-axis, whereas in $1 \cdot D M F$, the $X_{1}$-axis shows the largest thermal expansion coefficient among three principal axes, meaning that the thermal expansion of the guest dimer can change the main thermal expansion axis or thermal expansion anisotropy of the crystal. Anisotropic physical property, as one of the most important features of crystalline materials, is predominantly determined by the crystallographic symmetry. The guest-dependent anisotropy of thermal expansion of isomorphic 1, 1.DMF and 1.DMA (same crystallographic symmetry) demonstrates the possibility of controlling and utilizing the host-guest interplay for developing multiresponsive smart materials.

\section{Discussion}

In summary, by using mixed linear and bent ligands, we successfully constructed an ultramicroporous flexible framework with a highly connected $3 \mathrm{D}$ hinge-like structure and multimode flexibility, which exhibits the largest PTE and NTE coefficients among framework solids. Remarkably, the quasi-0D ultramicropores in this material can accommodate dimers of different small amide molecules, giving unprecedentedly significant guestmodulation effects on both the magnitude and anisotropy of thermal expansion. By virtue of the robustness, low symmetry and suitable pore size of the crystal, the guest- and temperatureinduced structural transformations were directly visualized by PXRD and SCXRD, which revealed that the confined guest dimers can show anisotropic thermal expansion behaviours similar with their bulk forms and force the host framework to drastically change not only the coefficients but also the main axis of thermal expansion. Generally, since the structural transformations of the host framework and the guest aggregations are always observed simultaneously, there is still no rational approach to analyse their relationship or identify which one is the original force. In this context, the multimode framework flexibility of the host framework, which has potential to deform in different ways under different stimuli, is crucial for unambiguous elucidation of the mechanism. Of course, these supramolecular-jack-like dimers and similar guest aggregations should be also effective and applicable for other flexible porous solids, even with only singlemode flexibility.

\section{Methods}

Materials. The ligands 3-(pyridin-4-yl)benzoic acid (H34pba) and 4-(pyridin4-yl)benzoic acid (H44pba) were synthesized according to literature ${ }^{17,32}$. Other reagents were commercially available and used without further purification.

Measurements. Elemental analyses were performed using a Vario EL

elemental analyser. The FT-IR (KBr pellet) spectra were recorded in the range of $400-4,000 \mathrm{~cm}^{-1}$ on a Bruker TENSOR 27 FT-IR spectrometer. Thermogravimetric analyses were carried out using a TA Instruments Q50 thermogravimetric analyser under $\mathrm{N}_{2}$ at a rate of $10^{\circ} \mathrm{C} / \mathrm{min}$. PXRD patterns were recorded on a Bruker D8Advance diffractometer using $\mathrm{Cu} \mathrm{K} \alpha$ radiation and a LynxEye detector at room temperature except otherwise stated. Gas sorption isotherms were measured on a volumetric adsorption apparatus (Micromeritics ASAP $2020 \mathrm{M}$ Physisorption Analyzer). Ultrahigh-purity-grade (purity >99.999\%) $\mathrm{N}_{2}, \mathrm{O}_{2}$ and $\mathrm{Ar}$ gases were used in all adsorption measurements. As-synthesized samples were degassed under high vacuum at $493 \mathrm{~K}$ for $3 \mathrm{~h}$ before measurement. Temperatures were maintained by liquid nitrogen.

Synthesis. H34pba $(0.40 \mathrm{~g}, 2.0 \mathrm{mmol})$ and H44pba $(0.40 \mathrm{~g}, 2.0 \mathrm{mmol})$ were dissolved in $\mathrm{EtOH}(80 \mathrm{ml})$ using a $250-\mathrm{ml}$ scintillation vial, added with a water solution of $\mathrm{Cd}\left(\mathrm{NO}_{3}\right)_{2}\left(0.05 \mathrm{moll}^{-1}, 40 \mathrm{ml}\right)$. The mixture was then sealed with a screw cap and heated to $90{ }^{\circ} \mathrm{C}$ for $24 \mathrm{~h}$. Colourless needle-like crystals of 1. EtOH $\cdot \mathrm{H}_{2} \mathrm{O}$ were obtained (yielded $0.81 \mathrm{~g}, \sim 80 \%$ ). Then the samples were filtered and briefly dried in air to give crystals of 1 . Elemental analysis calcd (\%) for [Cd(34pba)(44pba)] $3 \mathrm{H}_{2} \mathrm{O}\left(\mathrm{C}_{24} \mathrm{H}_{21} \mathrm{~N}_{2} \mathrm{O}_{7} \mathrm{Cd}\right): \mathrm{C} 51.31, \mathrm{H} 3.77, \mathrm{~N} 4.99$; found: $\mathrm{C}$ 51.20, H 4.35, N 4.68. FT-IR (KBr, cm $\left.{ }^{-1}\right): 3,426(\mathrm{~m}), 3,062(\mathrm{w}), 2,920(\mathrm{w}), 1,610(\mathrm{~s})$, 1,556(s), 1,396(s), 1,276(w), 1,224(w), 1,072(w), 1,014(w), 858(m), 837(m), 765(m), $736(\mathrm{~m}), 678(\mathrm{~m}), 624(\mathrm{w}), 559(\mathrm{w})$ and $503(\mathrm{~m})$. When single crystals of 1 were sealed in a test tube saturated with DMF/DMA vapour for 3 days, some single crystals of 1.DMF/1.DMA were obtained.

X-ray single-crystal structure analyses. Diffraction data were collected on an Agilent SuperNova CCD diffractometer with graphite-monochromated $\mathrm{Cu} \mathrm{K} \alpha$ radiation. Single crystals of $\mathbf{1} \cdot \mathrm{DMF}$ and $\mathbf{1}$. DMA were mounted directly on the top of a glass fibre using minimum amount of glue. A single crystal of $\mathbf{1}$ was sealed in high vacuum in a glass capillary. The test temperature was controlled by dry nitrogen flow using a Cryostream Plus cooler system, and corrected by a thermal couple at the crystal position. Though thermogravimetry curves of 1.DMF and 1.DMA showed no weight loss below $350 \mathrm{~K}$, the unit-cell parameters of 1.DMF and 1.DMA did change above $340 \mathrm{~K}$ under the temperature-controlling nitrogen flow after $\sim 2 \mathrm{~h}$, which can be attributed to the guest escaping. Nevertheless, under the same measurement conditions, no change of unit-cell parameter or chemical composition occurred for single crystals of 1.DMF and 1.DMA below $300 \mathrm{~K}$ even for one day. The unit-cell parameters at each measurement temperature used for calculation of the thermal expansion coefficients were determined by 30 diffraction images, while those for crystal-structure refinements were determined by 600-900 diffraction images. The reversibility of temperature-induced crystal deformations of the samples were confirmed by the coincidence of data points over a heatingcooling cycle. Absorption corrections were applied by using the multi-scan programme CrysAlisPro ${ }^{57}$. The crystal structures were solved through direct methods and developed by difference Fourier techniques using the SHELXTL software packages ${ }^{58}$. All hydrogen atoms were added geometrically, and anisotropic displacement parameters were used to refine all non-hydrogen atoms.

\section{References}

1. Horike, S., Shimomura, S. \& Kitagawa, S. Soft porous crystals. Nat. Chem. 1, 695-704 (2009).

2. Serre, C. et al. Role of solvent-host interactions that lead to very large swelling of hybrid frameworks. Science 315, 1828-1831 (2007).

3. Zhang, J. P. \& Chen, X. M. Exceptional framework flexibility and sorption behavior of a multifunctional porous cuprous triazolate framework. J. Am. Chem. Soc. 130, 6010-6017 (2008).

4. Warren, J. E. et al. Shape selectivity by guest- driven restructuring of a porous material. Angew. Chem. Int. Ed. 53, 4592-4596 (2014).

5. Keene, T. D. et al. Solvent-modified dynamic porosity in chiral 3D kagome frameworks. Dalton Trans. 42, 7871-7879 (2013).

6. Nagarkar, S. S., Desai, A. V. \& Ghosh, S. K. Stimulus-responsive metal-organic frameworks. Chem. Asian J. 9, 2358-2376 (2014).

7. Sakata, Y. et al. Shape-memory nanopores induced in coordination frameworks by crystal downsizing. Science 339, 193-196 (2013).

8. Yanai, N. et al. Guest-to-host transmission of structural changes for stimuliresponsive adsorption property. J. Am. Chem. Soc. 134, 4501-4504 (2012). 
9. Park, J. et al. Reversible alteration of $\mathrm{CO}_{2}$ adsorption upon photochemical or thermal treatment in a metal-organic framework. J. Am. Chem. Soc. 134, 99-102 (2012).

10. Henke, S., Schneemann, A. \& Fischer, R. A. Massive anisotropic thermal expansion and thermo-responsive breathing in metal-organic frameworks modulated by linker functionalization. Adv. Funct. Mater. 23, 5990-5996 (2013).

11. Rowsell, J. L. C., Spencer, E. C., Eckert, J., Howard, J. A. K. \& Yaghi, O. M. Gas adsorption sites in a large-pore metal-organic framework. Science 309, 1350-1354 (2005).

12. Dubbeldam, D., Walton, K. S., Ellis, D. E. \& Snurr, R. Q. Exceptional negative thermal expansion in isoreticular metal-organic frameworks. Angew. Chem. Int. Ed. 46, 4496-4499 (2007).

13. Wu, Y. et al. Negative thermal expansion in the metal-organic framework material $\mathrm{Cu}_{3}(1,3,5 \text {-benzenetricarboxylate) })_{2}$. Angew. Chem. Int. Ed. 47, 8929-8932 (2008)

14. Wu, Y., Peterson, V. K., Luks, E., Darwish, T. A. \& Kepert, C. J. Interpenetration as a mechanism for negative thermal expansion in the metal-organic framework $\mathrm{Cu}_{3}(\mathrm{btb})_{2}$ (MOF-14). Angew. Chem. Int. Ed. 53, 5175-5178 (2014).

15. DeVries, L. D., Barron, P. M., Hurley, E. P., Hu, C. H. \& Choe, W. 'Nanoscale lattice fence' in a metal-organic framework: interplay between hinged topology and highly an isotropic thermal response. J. Am. Chem. Soc. 133, 14848-14851 (2011).

16. Wei, Y.-S. et al. Turning on the flexibility of isoreticular porous coordination frameworks for drastically tunable framework breathing and thermal expansion. Chem. Sci. 9, 1539-1546 (2013).

17. Zhou, H. L. et al. Direct visualization of a guest-triggered crystal deformation based on a flexible ultramicroporous framework. Nat. Commun. 4, 2534 (2013).

18. Grobler, I., Smith, V. J., Bhatt, P. M., Herbert, S. A. \& Barbour, L. J. Tunable anisotropic thermal expansion of a porous zinc(II) metal-organic framework. J. Am. Chem. Soc. 135, 6411-6414 (2013).

19. Yang, C., Wang, X. P. \& Omary, M. A. Crystallographic observation of dynamic gas adsorption sites and thermal expansion in a breathable fluorous metalorganic framework. Angew. Chem. Int. Ed. 48, 2500-2505 (2009).

20. Cai, W. \& Katrusiak, A. Giant negative linear compression positively coupled to massive thermal expansion in a metal-organic framework. Nat. Commun. 5, 4337 (2014).

21. Mary, T. A., Evans, J. S. O., Vogt, T. \& Sleight, A. W. Negative thermal expansion from 0.3 to 1050 Kelvin in $\mathrm{ZrW}_{2} \mathrm{O}_{8}$. Science 272, 90-92 (1996).

22. Evans, J. S. O. Negative thermal expansion materials. J. Chem. Soc., Dalton Trans. 3317-3326 (1999).

23. Barrera, G. D., Bruno, J. A. O., Barron, T. H. K. \& Allan, N. L. Negative thermal expansion. J. Phys. Condens. Mat 17, R217-R252 (2005).

24. Han, S. S. \& Goddard, W. A. Metal-organic frameworks provide large negative thermal expansion behavior. J. Phys. Chem. C 111, 15185-15191 (2007).

25. Goodwin, A. L. et al. Colossal positive and negative thermal expansion in the framework material $\mathrm{Ag}_{3}\left[\mathrm{Co}(\mathrm{CN})_{6}\right]$. Science 319, 794-797 (2008).

26. Das, D., Jacobs, T. \& Barbour, L. J. Exceptionally large positive and negative anisotropic thermal expansion of an organic crystalline material. Nat. Mater. 9, 36-39 (2010).

27. Panda, M. K. et al. Colossal positive and negative thermal expansion and thermosalient effect in a pentamorphic organometallic martensite. Nat. Commun. 5, 4811 (2014).

28. Deng, H. X., Olson, M. A., Stoddart, J. F. \& Yaghi, O. M. Robust dynamics. Nat. Chem. 2, 439-443 (2010).

29. Browne, W. R. \& Feringa, B. L. Making molecular machines work. Nat. Nanotechnol. 1, 25-35 (2006).

30. Wang, C., Zhang, T. \& Lin, W. Rational synthesis of noncentrosymmetric metal-organic frameworks for second-order nonlinear optics. Chem. Rev. 112, 1084-1104 (2012).

31. Zhang, Z. J., Wojtas, L. \& Zaworotko, M. J. Organic-inorganic hybrid polyhedra that can serve as supermolecular building blocks. Chem. Sci. 5, 927-931 (2014).

32. Zhang, Y. B. et al. Geometry analysis and systematic synthesis of highly porous isoreticular frameworks with a unique topology. Nat. Commun. 3, 642 (2012).

33. Collings, I. E. et al. Homologous critical behavior in the molecular frameworks $\mathrm{Zn}(\mathrm{CN})_{2}$ and Cd(imidazolate) $)_{2} . J$. Am. Chem. Soc. 135, 7610-7620 (2013)

34. Collings, I. E., Tucker, M. G., Keen, D. A. \& Goodwin, A. L. Geometric switching of linear to area negative thermal expansion in uniaxial metal-organic frameworks. CrystEngComm 16, 3498-3506 (2014).

35. van Oosten, C. L., Bastiaansen, C. W. M. \& Broer, D. J. Printed artificial cilia from liquid-crystal network actuators modularly driven by light. Nat. Mater. 8, 677-682 (2009).

36. Halder, G. J., Kepert, C. J., Moubaraki, B., Murray, K. S. \& Cashion, J. D. Guest-dependent spin crossover in a nanoporous molecular framework material. Science 298, 1762-1765 (2002).
37. Takashima, Y. et al. Molecular decoding using luminescence from an entangled porous framework. Nat. Commun. 2, 168 (2011).

38. Talin, A. A. et al. Tunable electrical conductivity in metal-organic framework thin-film devices. Science 343, 66-69 (2014).

39. Zhao, H. X. et al. Transition from one-dimensional water to ferroelectric ice within a supramolecular architecture. Proc. Natl Acad. Sci. USA 108, 3481-3486 (2011).

40. Goodwin, A. L., Chapman, K. W. \& Kepert, C. J. Guest-dependent negative thermal expansion in nanoporous Prussian Blue analogues $\mathrm{M}^{\mathrm{II}} \mathrm{Pt}^{\mathrm{IV}}(\mathrm{CN})_{6} \cdot x\left\{\mathrm{H}_{2} \mathrm{O}\right\}(0 \leq \mathrm{x} \leq 2 ; \mathrm{M}=\mathrm{Zn}, \mathrm{Cd})$. J. Am. Chem. Soc. 127, 17980-17981 (2005).

41. Phillips, A. E., Goodwin, A. L., Halder, G. J., Southon, P. D. \& Kepert, C. J. Nanoporosity and exceptional negative thermal expansion in single-network cadmium cyanide. Angew. Chem. Int. Ed. 47, 1396-1399 (2008).

42. Kitaura, R. et al. Formation of a one-dimensional array of oxygen in a microporous metal-organic solid. Science 298, 2358-2361 (2002).

43. Zhang, J. P., Liao, P. Q., Zhou, H. L., Lin, R. B. \& Chen, X. M. Single-crystal $\mathrm{X}$-ray diffraction studies on structural transformations of porous coordination polymers. Chem. Soc. Rev. 43, 5789-5814 (2014).

44. Vaidhyanathan, R. et al. Direct observation and quantification of $\mathrm{CO}_{2}$ binding within an amine-functionalized nanoporous solid. Science 330, 650-653 (2010).

45. Shinohara, K. et al. A chiral wedge molecule inhibits telomerase activity. J. Am Chem. Soc. 132, 3778-3782 (2010).

46. Kawamichi, T., Haneda, T., Kawano, M. \& Fujita, M. X-ray observation of a transient hemiaminal trapped in a porous network. Nature 461, 633-635 (2009).

47. Inokuma, Y. et al. X-ray analysis on the nanogram to microgram scale using porous complexes. Nature 495, 461-466 (2013).

48. Chen, B. L., Xiang, S. C. \& Qian, G. D. Metal-organic frameworks with functional pores for recognition of small molecules. Acc. Chem. Res. 43, 1115-1124 (2010).

49. Lin, J. B. et al. Chemical/physical pressure tunable spin-transition temperature and hysteresis in a two-step spin crossover porous coordination framework. Inorg. Chem. 51, 9423-9430 (2012).

50. Long, D. L. et al. Non-natural eight-connected solid-state materials: a new coordination chemistry. Angew. Chem. Int. Ed. 43, 1851-1854 (2004).

51. Long, D. L., Blake, A. J., Champness, N. R., Wilson, C. \& Schroder, M. Unprecedented seven- and eight-connected lanthanide coordination networks. Angew. Chem. Int. Ed. 40, 2444-2513 (2001).

52. Mehlana, G., Bourne, S. A., Ramon, G. \& Ohrstrom, L. Concomitant metal organic frameworks of cobalt(II) and 3-(4-Pyridyl)benzoate: optimized synthetic conditions of solvatochromic and thermochromic systems. Cryst. Growth Des. 13, 633-644 (2013).

53. Ogborn, J. M., Collings, I. E., Moggach, S. A., Thompson, A. L. \& Goodwin, A L. Supramolecular mechanics in a metal-organic framework. Chem. Sci. 3, 3011-3017 (2012).

54. Wernsdorfer, W., Aliaga-Alcalde, N., Hendrickson, D. N. \& Christou, G. Exchange-biased quantum tunnelling in a supramolecular dimer of singlemolecule magnets. Nature 416, 406-409 (2002).

55. Yamauchi, K., Takashima, Y., Hashidzume, A., Yamaguchi, H. \& Harada, A. Switching between supramolecular dimer and nonthreaded supramolecular self-assembly of stilbene amide- $\alpha$-cyclodextrin by photoirradiation. J. Am. Chem. Soc. 130, 5024-5025 (2008).

56. Cliffe, M. J. \& Goodwin, A. L. PASCal: a principal axis strain calculator for thermal expansion and compressibility determination. J. Appl. Cryst. 45, 1321-1329 (2012).

57. CrysAlisPRO, Oxford Diffraction. (Agilent, Technologies, UK Ltd, West Lothian, UK, 2011)

58. Sheldrick, G. M. SHELX-97: Programs for Crystal Structure Analysis (University of Göttingen, 1997).

\section{Acknowledgements}

This work was supported by the '973 Project' (2012CB821706 and 2014CB845602) and NSFC $(21225105,21290173$ and 21473260).

\section{Author contributions}

J.P.Z. designed the research; H.L.Z. performed synthesis and measurements; J.P.Z. and H.L.Z. analysed data; all authors discussed the results, contributed to the writing of manuscript and commented on it.

\section{Additional information}

Accession codes. The X-ray crystallographic coordinates for structures reported in this Article have been deposited at the Cambridge Crystallographic Data Centre (CCDC), 
under deposition number CCDC 1049147-1049152. These data can be obtained free of charge from The Cambridge Crystallographic Data Centre via www.ccdc.cam.ac.uk/ data_request/cif.

Supplementary Information accompanies this paper at http://www.nature.com/ naturecommunications

Competing financial interests: The authors declare no competing financial interests.

Reprints and permission information is available at http://npg.nature.com/ reprintsandpermissions/
How to cite this article: Zhou, H.-L. et al. Supramolecular-jack-like guest in ultramicroporous crystal for exceptional thermal expansion behaviour. Nat. Commun. 6:6917 doi: 10.1038/ncomms7917 (2015).

(c) (i) This work is licensed under a Creative Commons Attribution 4.0 International License. The images or other third party material in this article are included in the article's Creative Commons license, unless indicated otherwise in the credit line; if the material is not included under the Creative Commons license, users will need to obtain permission from the license holder to reproduce the material. To view a copy of this license, visit http://creativecommons.org/licenses/by/4.0/ 